\title{
Identification of primary care patients at risk of nonadherence to antidepressant treatment
}

\author{
Ann-Charlotte Åkerblad' \\ Finn Bengtsson ${ }^{2}$ \\ Margareta Holgersson ${ }^{3}$ \\ Lars von Knorring' \\ Lisa Ekselius' \\ 'Department of Neuroscience, \\ Psychiatry, Uppsala University \\ Hospital, Uppsala University, Uppsala, \\ Sweden; ${ }^{2}$ Division of Clinical \\ Pharmacology, Medicine and Care, \\ Faculty of Health Sciences, Linköping \\ University, Linköping, Sweden; \\ ${ }^{3}$ Quintiles AB, Uppsala, Sweden
}

\begin{abstract}
Introduction: Poor adherence to antidepressant treatment is common, and results in increased disability and costs. Several factors are thought to influence patients' ability and willingness to adhere. So far, however, consensus is lacking regarding patient characteristics that predict nonadherence. The purpose of this study was to identify predictors of nonadherence to antidepressant treatment that can be ascertained at treatment start.

Method: The present study used data from a randomized controlled trial with the main objective of studying the effect of two different compliance-enhancing programs on treatment adherence and treatment response in 1031 primary care patients with major depression. In this study, logistic regression analyses were performed to examine patient- and illness-related characteristics potentially associated with nonadherence.

Results: Nonadherence to antidepressant treatment was predicted by age under 35 or over 64 years, presence of personality disorder, sensation-seeking personality traits, substance abuse, and absence of concomitant medications.

Conclusion: Certain patient- and illness-related characteristics may imply an increased risk of nonadherence to antidepressant treatment. Giving special attention to subjects with such characteristics may improve adherence.
\end{abstract}

Keywords: unipolar depression, antidepressant, adherence, compliance, SSRI, predictors

\section{Introduction}

Adherence to antidepressant medication has been recognized as an important factor regarding optimal treatment outcome (Frank et al 1992; Melfi et al 1998; Åkerblad et al 2006; Gopinath et al 2007). Nonadherence behavior includes premature or temporary discontinuation of medication, erratic intake or intake of a lower or higher dose than prescribed. Despite treatment recommendations, it is probable that less than half of primary care patients diagnosed with depression and prescribed antidepressants take their medication for six to nine months or longer (Demyttenaere et al 2001; Cantrell et al 2006; Olfson et al 2006). In a large retrospective study of 22,947 patients starting treatment with selective serotonin reuptake inhibitors (SSRIs), the six-month nonadherence rate was approximately $57 \%$ as measured by the length of treatment and medication possession ratio (Cantrell et al 2006). When the patients' partial or erratic adherence to treatment is taken into account, the proportion of patients with adequate treatment probably decreases further. Overall, nonadherence to antidepressant drug treatment has been found to vary from 10\% to $60 \%$ (Lingam and Scott 2002; Åkerblad et al 2003). Another important aspect of antidepressant treatment is clinic visits (Demyttenaere 2003). A relation between adherence to the clinic visit-schedule and outcome has been shown ( $\AA$ kerblad et al 2003).

The ability of patients to follow prescribed treatment is often hindered by different factors including social and economic circumstances, the health care system, the provider, the disease and its treatment, and patient-related factors (Demyttenaere 1997; Osterberg and Blaschke 2005). Identifying subjects at risk of nonadherence
Correspondence:Ann-Charlotte Åkerblad Department of Neuroscience, Psychiatry, Uppsala University Hospital, Uppsala University, SE-75I 85 Uppsala, Sweden Emailann-charlotte.akerblad@uaspsyk.uu.se 
right from the start of treatment increases the possibility of intervening with the aim of improving adherence. This early identification of patients at risk of nonadherence might thus improve the chance of optimal treatment and a better overall outcome. It cannot be ruled out that premature dropout, nonadherence and nonattendance are, at least in part, predicted by different characteristics as suggested by Demyttenaere and colleagues (1998). However, regardless of the nature of nonadherence to treatment behavior, a relation between adherence and outcome has been shown ( $\AA$ kerblad et al 2003). Research on easily identified predictors of nonadherence to antidepressant drug treatment such as demographics, sociodemographics, illness characteristics and personality is limited, rather inconclusive, and involves small numbers of patients (Pampallona et al 2002; Demyttenaere 2003; Cohen et al 2004). In the present study an attempt was made to identify predictors of nonadherence to antidepressants that can be ascertained by the physician before initiation of treatment. We hypothesized that demographics, depression characteristics, and personality are of importance regarding poor adherence.

\section{Subjects and methods Study sample}

Data for this study were obtained from a randomized, controlled, multicenter study, the Swedish Long-term Implications of Compliance Enhancing programs study (SLICE) (Åkerblad et al 2003, 2006). The main objective of that investigation was to study the effect of two different compliance-enhancing programs on treatment adherence and treatment response to sertraline during a 24-week period. Patients in the Compliance Enhancement Program (CP) group were provided with written educational material covering typical issues and recovery patterns associated with successful treatment of major depression (Mundt et al 2001; Kutcher et al 2002). The goal of the program is to maximize the therapeutic outcome by encouraging adherence to treatment. A starter kit was given to the patient when treatment began, and a total of five different letters were mailed to the patient during the first 18 weeks of treatment. In the Therapeutic Drug Monitoring (TDM) group, blood samples from the patients were collected for immediate analysis of sertraline and the major metabolite, desmethylsertraline, at weeks 4 and 12 . The physician responsible for treatment and the TDM laboratory communicated via standardized request forms. By means of TDM, dose optimization for individual patients, including monitoring of adherence, side effects and drug-drug interactions, can be accomplished (Burke and Preskorn 1999; Bengtsson 2004).
The two intervention groups were compared with a control group in which patients were treated in accordance with the general practitioners' clinical routine. Neither of the two interventions tested improved the adherence rate significantly as compared to the control group (Åkerblad et al 2003). The study methods and the results for the initial 24 weeks were previously presented in detail (Åkerblad et al 2003).

The SLICE-study was conducted in primary care with general practitioners managing the treatment. A total of 93 general practitioners included patients in the open-labeled study. In order to avoid carrying over some of the features of the different adherence interventions between treatment groups, a cluster randomization design was used. Thus, the general practitioners, and not the patients, were randomized to the three treatment groups, and each practitioner included patients in one treatment group only. The randomization was done in a consecutive way based on a computerized randomization list.

To assess adherence and clinical outcome, visits at the outpatient department were conducted at baseline, and at 4, 12, and 24 weeks. Data were collected by the general practitioners on case report forms (CRFs) designed for this study. During the study, concordance between the medical records and the case report forms was monitored.

To be included in the study, patients had to fulfill the criteria for major depression according to the DSM-IV (APA 1994) and have a clinical indication for treatment with an SSRI. Male and female patients 18 years of age or older were included if they gave their written informed consent to participate in the study. The only exclusion criteria were contraindications to sertraline.

The study was conducted in accordance with the Declaration of Helsinki (Revised in South Africa in 1996). It was approved by the regional ethics committees at the Swedish universities in Umeå, Uppsala, Örebro, Stockholm, Linköping, and Gothenburg.

\section{Assessments}

\section{Measurement of nonadherence}

In the present study, a conservative definition of adherence was used (Åkerblad et al 2003) requiring: (a) measurable serum levels of sertraline and/or desmethylsertraline at weeks 4, 12 and 24 (ie, blood samples were taken in all three groups, but during the study the results were disclosed only in the TDM-group); (b) self-reported assurance that the patient had taken sertraline as prescribed. (The patient was asked by the general practitioner if he/she had taken the medication as prescribed. Based on the patient's answer the 
general practitioner recorded a "Yes" or a "No" on the CRF); and (c) scheduled visits performed within the stipulated time frames (ie, $4 \pm 1,12 \pm 4$, and $24 \pm 2$ weeks). Accordingly, patients discontinuing sertraline treatment prematurely, withdrawing from the study, or not fulfilling one or more of the three adherence criteria specified above were classified as nonadherent at 24 weeks. As adherence is then defined and used as a dichotomous variable, adherence vs nonadherence, patients with nonadherence are all patients who do not fulfill the adherence criteria, thus comprising a somewhat heterogeneous group.

\section{Personality disorders and personality traits}

The presence of personality disorders was assessed using the DSM-IV and ICD-10 Personality Questionnaire (DIP-Q). This is a 135-item true/false self-report questionnaire designed to measure personality disorders according to DSM-IV and ICD-10. The DIP-Q has been validated by comparing questionnaire-based results with those from a semi-structured interview in a clinical sample of 138 individuals (Ottoson et al 1998). In the present study only DSM-IV personality disorders were assessed, and anti-social personality was excluded.

Personality traits were assessed by the Swedish Universities Scales of Personality (SSP) (Gustavsson et al 2000). The SSP comprises 91 items divided into 13 scales with seven items in each. Each item is presented as a statement with a four-point response format, ranging from 1 , "does not apply at all" to 4, "applies completely". The SSP mean scores were transformed into normative $\mathrm{T}$-scores with means of 50 and standard deviations of 10 based on a Swedish gender-stratified nonpatient sample (Gustavsson et al 2000). A maximum likelihood factor analysis with orthogonal rotation (varimax) was performed to identify factors with an eigenvalue $>1$. Similar to the factor analysis of the normative data (Gustavsson et al 2000), the correlations of the 13 scales yielded a three-factor model. The scales Somatic Trait Anxiety, Psychic Trait Anxiety, Stress Susceptibility, Lack of Assertiveness, Detachment, Embitterment, Trait Irritability, and Mistrust loaded highly on the first factor, hereafter referred to as Neuroticism. The second factor consisted of Social Desirability (negative loading), Verbal Trait Aggression, Trait Irritability, and Physical Trait and will be referred to as Aggressiveness. Impulsiveness and Adventure Seeking loaded highly on the third factor, termed Sensation Seeking. The corresponding scores from the three factors were subsequently used in the statistical analyses.

\section{Quality of life}

Health-related quality of life was assessed at baseline by the EuroQol questionnaire - 5 dimensions (EQ-5D) (EuroQoL Group 1990). The EQ-5D is a standardized and widely used generic instrument comprised of two parts: a visual analogue scale, and five questions that assess the respondent's health according to five distinct dimensions: mobility, self-care, usual activities, pain/discomfort, and anxiety/depression. Each dimension has three different levels: no problems, some/ moderate problems, and extreme problems. Combined, these dimensions and levels make up 243 unique theoretical health states, to which quality of life index scores between -0.59 and 1 (full health), also referred to as societal valuation, were assigned using a standardized tariff based on the general UK population (Dolan et al 1996). The results from the visual analogue scale were not used in the present study.

\section{Severity of depression}

The severity of depression was assessed by means of the Montgomery-Åsberg Depression Rating Scale (MADRS) (Montgomery and Åsberg 1979) and the Clinical Global Impression - Severity scale (CGI-S) (Guy 1976). Before the start of the trial, a weighted kappa of 0.7 for inter-rater reliability of MADRS ratings was obtained.

\section{Potentially associated predictors}

Predictor variables potentially associated with adherence to antidepressants were selected based on earlier research and clinical experience (Frank et al 1992; Lin et al 1995; Demyttenaere et al 1998; Ekselius et al 2000; Cohen et al 2004). The variables were grouped into patient-related characteristics and illness-related characteristics. The variables are shown in Tables 1 and 2 .

\section{Statistics}

All statistical analyses were performed using SAS ${ }^{\circledR}$ software (version 8.2; SAS Institute, Cary, NC). Data presented in this report are based on the intention-to-treat (ITT) population.

In summary, the statistical analyses in the present study included bivariate evaluation of the relationship between nonadherence and each potential predictor, followed by a stepwise logistic regression analysis.

Prior to the bivariate analyses, all continuous variables were inspected with regard to conformity with a linear gradient. Based on the finding of a U-formed relation with nonadherence seen with increasing age when plotting the proportion of nonadherent patients for different age groups, the variable age was categorized into three groups (18-34 years, 35-64 years, and 
Table I Associations between patient-related characteristics and adherence

\begin{tabular}{|c|c|c|c|}
\hline Variable & Adherent & Nonadherent & $\mathbf{p}$ \\
\hline \multicolumn{4}{|l|}{ Gender, n (\%) } \\
\hline Female & $308(4 \mid .6)$ & $433(58.4)$ & \multirow[t]{2}{*}{$0.29^{\prime}$} \\
\hline Male & $110(37.9)$ & $180(62.1)$ & \\
\hline \multicolumn{4}{|l|}{ Age, n (\%) } \\
\hline Low: 18-34 yrs & $54(26.7)$ & $148(73.3)$ & \multirow[t]{3}{*}{$<0.000 I^{\prime}$} \\
\hline (reference) Mid: 35-64 yrs & $319(45.3)$ & $385(54.7)$ & \\
\hline High: $\geq 65$ yrs & $45(36.0)$ & $80(64.0)$ & \\
\hline \multicolumn{4}{|l|}{ Living status, n (\%) } \\
\hline Married/cohabiting & $273(43.1)$ & $360(56.9)$ & \multirow[t]{2}{*}{$0.03 I^{\prime}$} \\
\hline Other & $145(36.4)$ & $253(63.6)$ & \\
\hline \multicolumn{4}{|l|}{ at home, mean $(S D)^{3}$} \\
\hline \multicolumn{4}{|l|}{ Education, n (\%) } \\
\hline Primary school & $|3|(42.3)$ & $179(57.7)$ & \multirow[t]{3}{*}{$0.92^{\prime}$} \\
\hline (reference) High school & I $88(4 \mid .2)$ & $268(58.8)$ & \\
\hline University & $86(40.6)$ & $126(59.4)$ & \\
\hline \multicolumn{4}{|l|}{ Employment, n (\%) } \\
\hline -yes & $297(42.8)$ & $397(57.2)$ & \multirow[t]{2}{*}{$0.035^{\prime}$} \\
\hline - other & 121 (35.9) & $216(64.1)$ & \\
\hline \multicolumn{4}{|l|}{ Student, n (\%) } \\
\hline- yes & $19(29.7)$ & $45(70.3)$ & \multirow[t]{2}{*}{$0.068^{\prime}$} \\
\hline - other & $399(4 I .3)$ & $568(58.7)$ & \\
\hline \multicolumn{4}{|l|}{ Unemployment, n (\%) } \\
\hline- yes & $30(34.1)$ & $58(65.9)$ & \multirow[t]{2}{*}{$0.20^{\prime}$} \\
\hline - other & $388(4 \mid .2)$ & $555(58.8)$ & \\
\hline \multicolumn{4}{|l|}{ Retired, n (\%) } \\
\hline - yes & $46(36.2)$ & $81(63.8)$ & \multirow[t]{2}{*}{$0.29^{\prime}$} \\
\hline - other & $372(4 I .2)$ & $532(58.9)$ & \\
\hline \multicolumn{4}{|l|}{ Early retirement, n (\%) } \\
\hline- yes & $30(44.1)$ & $38(55.9)$ & \multirow[t]{2}{*}{$0.53^{\prime}$} \\
\hline - other & $388(40.3)$ & $575(59.7)$ & \\
\hline \multicolumn{4}{|l|}{ Nicotine use, n (\%) } \\
\hline - yes & $150(37.2)$ & $253(62.8)$ & \multirow[t]{2}{*}{$0.082^{\prime}$} \\
\hline- no & $268(42.7)$ & $360(57.3)$ & \\
\hline Neuroticism factor score*, mean (SD) & $69.4(9.91)$ & $71.2(10.9)$ & $0.007 I^{2}$ \\
\hline Aggressiveness factor score*, mean (SD) & $20.4(8.83)$ & $21.8(9.91)$ & $0.022^{2}$ \\
\hline Sensation seeking factor score*, mean (SD) & $48.2(8.90)$ & $50.5(8.75)$ & $<0.0001^{2}$ \\
\hline
\end{tabular}

Notes: *Each estimated factor score is computed as a linear combination of the unstandardized values of the variables that are factored; 'Chi-square test; ${ }^{2} \mathrm{t}$-test; ${ }^{3} \mathrm{missing}$ data for one adherent patient and 30 nonadherent patients.

Abbreviation: SD, standard deviation.

65 years and older). In addition, due to a nonlinear relationship, "age at first depression" and "concomitant medication" were categorized in the subsequent analyses.

The bivariate analyses included all patient- and illnessrelated characteristics potentially associated with nonadherence as described above. Testing for association with nonadherence was performed using chi-square tests (categorical variables) or t-tests (continuous variables). Variables with a p-value of 0.25 or less were included in logistic regression analyses (Hosmer et al 2000) with nonadherence as the dependent variable $\left(\mathrm{SAS}^{\circledR}\right.$ PROC LOGISTIC). The variables were included in order of statistical significance starting with the variable with the lowest p-value. In order to adjust for the interventions in the analyses, this variable was included in the model although it did not meet the $p \leq 0.25$ criterion. The inclusion of intervention group in the model was expected to reduce the possible bias of pooling the three treatment groups.

In the regression analyses only variables remaining statistically significant $(\mathrm{p}<0.05)$ were retained in the model. 
Table 2 Associations between illness-related characteristics and adherence

\begin{tabular}{|c|c|c|c|}
\hline Variable & Adherent & Nonadherent & $\mathbf{p}$ \\
\hline \multicolumn{4}{|l|}{ Baseline characteristics } \\
\hline Total MADRS-score, mean (SD) & $26.5(5.65)$ & $27.0(5.97)$ & $0.12^{\prime}$ \\
\hline CGI-S-score, mean (SD) & $4.16(0.78)$ & $4.22(0.79)$ & $0.23^{\prime}$ \\
\hline QoL societal valuation, mean $(\mathrm{SD})^{3}$ & $0.61(0.27)$ & $0.60(0.27)$ & $0.53^{\prime}$ \\
\hline \multicolumn{4}{|l|}{ Personality disorder, n (\%) } \\
\hline- yes & $107(31.9)$ & $228(68.1)$ & \multirow[t]{2}{*}{$<0.000 \mathrm{I}^{2}$} \\
\hline- no & $310(46.5)$ & $357(53.5)$ & \\
\hline \multicolumn{4}{|l|}{ Concomitant medications, n (\%) } \\
\hline- yes & $31(44.1)$ & $420(55.9)$ & \multirow{2}{*}{$0.0002^{2}$} \\
\hline- no & $87(31.1)$ & $193(68.9)$ & \\
\hline \multicolumn{4}{|l|}{ Psychiatric history } \\
\hline \multicolumn{4}{|l|}{ Previous depression, $\mathrm{n}(\%)$} \\
\hline- yes & $25 I(42.0)$ & $346(58.0)$ & \multirow{2}{*}{$0.25^{2}$} \\
\hline- no & $167(38.5)$ & $267(6 \mid .5)$ & \\
\hline \multicolumn{4}{|l|}{ No of previous depression episodes } \\
\hline (if any), mean (SD) ${ }^{4}$ & $3.24(3.88)$ & $3.62(4.87)$ & 0.291 \\
\hline \multicolumn{4}{|l|}{ Age at first depression episode, $\mathrm{n}(\%)$} \\
\hline $0-29$ yrs & $103(34.2)$ & $198(65.8)$ & \multirow{3}{*}{$0.0073^{2}$} \\
\hline $30-64$ yrs (ref) & $287(44.2)$ & $362(55.8)$ & \\
\hline$\geq 65$ yrs & $28(34.6)$ & $53(65.4)$ & \\
\hline \multicolumn{4}{|l|}{ Hospitalized for depression, n (\%) } \\
\hline- yes & $32(45.1)$ & $39(54.9)$ & \multirow[t]{2}{*}{$0.42^{2}$} \\
\hline- no & $386(40.2)$ & $574(59.8)$ & \\
\hline \multicolumn{4}{|c|}{ Previous antidepressant drug treatment, $\mathrm{n}(\%)$} \\
\hline- yes & $182(43.3)$ & $238(56.7)$ & \multirow[t]{2}{*}{$0.13^{2}$} \\
\hline- no & $236(38.6)$ & $375(6 \mid .4)$ & \\
\hline \multicolumn{4}{|c|}{ Successful prev. antidepressant treatment, n (\%) } \\
\hline- yes & $90(46.6)$ & $103(53.4)$ & \multirow{2}{*}{$0.056^{2}$} \\
\hline- no & $328(39.1)$ & $510(60.9)$ & \\
\hline \multicolumn{4}{|l|}{ Suicide attempt(s) in the past, $\mathrm{n}(\%)$} \\
\hline- yes & $26(35.1)$ & $48(64.9)$ & \multirow{2}{*}{$0.33^{2}$} \\
\hline- no & $392(41.0)$ & $565(59.0)$ & \\
\hline \multicolumn{4}{|l|}{ Other psychiatric illness (past), n (\%) } \\
\hline- yes & $50(44.3)$ & $63(55.7)$ & \multirow{2}{*}{$0.40^{2}$} \\
\hline- no & $368(40.1)$ & $550(59.9)$ & \\
\hline \multicolumn{4}{|l|}{ Other psych. illness (present), n (\%) } \\
\hline- yes & $40(38.1)$ & $65(61.9)$ & \multirow{2}{*}{$0.59^{2}$} \\
\hline- no & $378(40.8)$ & $548(59.2)$ & \\
\hline \multicolumn{4}{|l|}{ Substance abuse (past/present), n (\%) } \\
\hline- yes & $20(25.0)$ & $60(75.0)$ & \multirow{2}{*}{$0.0032^{2}$} \\
\hline- no & $398(41.8)$ & $553(58.2)$ & \\
\hline Depression in family, $\mathrm{n}(\%)$ & & & \\
\hline- yes & III (38.7) & $176(6 \mid .3)$ & 0412 \\
\hline- no & $307(4 \mid .3)$ & $437(58.7)$ & $0.43^{2}$ \\
\hline
\end{tabular}

Notes: ' $\mathrm{t}$-test; ${ }^{2} \mathrm{Chi}$-square test; ${ }^{3}$ Missing data for 13 adherent patients and 33 nonadherent patients; ${ }^{4} \mathrm{n}=248$ for adherent patients and $34 \mathrm{I}$ for nonadherent patients. Abbreviation: SD, standard deviation. 
Odds ratios (OR) were estimated with 95\% confidence intervals for these variables. The resulting logistic models were re-estimated by means of a generalized estimating equations model (Hardin and Hilbe 2002), using SAS ${ }^{\circledR}$ PROC GENMOD to adjust for the effects of the cluster randomization design.

Based on the protocol, all patients were requested to complete the DIP-Q and SSP questionnaires at baseline, but 70 and 73 patients, respectively, did not do so. In order to reduce the number of missing observations, imputation of the results from the personality assessment after 12 weeks was done for 41 subjects regarding the presence of any personality disorder, and for 42 subjects regarding the personality traits.

\section{Results}

\section{Study population}

The ITT population included 1031 patients $(71.9 \%$ females, $28.1 \%$ males) with a mean age of 48.4 years and a mean MADRS score at baseline of 26.8. The demographic profile of the study population was described in a previous report (Åkerblad et al 2003) In the regression analyses, data from 33 patients were deleted due to missing values.

\section{Nonadherence to treatment}

During the 24-week study period, 249 patients $(24.1 \%)$ discontinued treatment with sertraline and/or withdrew from the study. Of the 782 patients completing 24 weeks of treatment, 364 were classified as nonadherent; 141 (38.7\%) did not complete the questionnaires, $78(21.4 \%)$ did not have measurable serum levels, and $219(60.2 \%)$ did not adhere to the appointment schedule. Thus, $613(59.5 \%)$ of the 1031 patients did not fulfill the pre-defined adherence criteria.

\section{Patient- and illness-related characteristics}

Bivariate associations between patient- and illness-related characteristics and adherence are presented in Table 1 and Table 2, respectively. A total of 18 variables had a p-value of 0.25 or less and were accordingly included in the primary step-wise logistic regression analysis. After controlling for intervention group, the logistic regression analysis revealed that patients in the younger age group (18-34 years) and patients in the older age group (65 years and older), with the middle age group as reference, were more likely to be nonadherent (Table 3). Patients with no concomitant medication, all medications regardless of underlying condition included, were also more likely to be nonadherent. The analysis also showed that the presence of personality disorder, sensation-seeking personality traits and substance abuse each predicted nonadherence.

\section{Goodness-of-fit}

Goodness-of-fit was evaluated using Pearson's residual chi-square test. The null hypothesis of good fit (small discrepancies between observed and predicted values according to the model) could not be rejected, indicating a high goodness-of-fit. The test results were: chi-square $=7.53(\mathrm{df}=15)$, and $\mathrm{p}=0.94$.

\section{Discussion}

Predictors of poor adherence to antidepressant treatment can be a useful resource in identifying those patients who are most in need of interventions to improve adherence (Osterberg and Blaschke 2005). However, previous research is limited and has most often involved small numbers of patients (Pampallona et al 2002; Demyttenaere 2003). In the present study, low age as well as high age, presence of personality disorder, sensation-seeking personality traits, substance abuse, and absence of concomitant medication were shown to be independent predictors.

\section{Patient-related characteristics}

In our study, both young age and old age were shown to predict poor adherence. The results of earlier studies, where age was included as a potential predictor of adherence/nonadherence to antidepressants, are somewhat conflicting. In several studies, no relation between age and adherence was found (Frank et al 1992; Lin et al 1995; Bull et al 2002). However, in line with our finding, Demyttenaere and colleagues (1998) reported that young age was a predictor of premature dropout from antidepressant drug treatment. Our finding that high age predicts nonadherence is contradicted by the results of Sirey and colleagues (2001) showing that age over 60 years was a predictor for adherence. In previous research in other therapeutic areas, high age has not consistently been shown to predict either adherence or nonadherence, although some potentially age-related factors such as living situation and polypharmacy have been shown to be related to nonadherence

Table 3 Logistic regression estimates (odds ratios) for the effect of patient- and illness-related characteristics on nonadherence ${ }^{1}$

\begin{tabular}{lll}
\hline & OR & $95 \%$ CI \\
\hline Age I8-35 years vs 35-64 years & 1.80 & $1.29-2.53$ \\
65 years vs 35-64 years & 1.60 & $1.01-2.53$ \\
Alcohol/drug abuse (past and/or present) & 1.80 & $1.07-3.05$ \\
Absence of concomitant medication & 1.79 & $1.32-2.44$ \\
Presence of personality disorder & 1.43 & $1.10-1.87$ \\
Sensation-seeking personality traits & 1.25 & $1.10-1.43$ \\
factor score (unit = I SD) & & \\
\hline
\end{tabular}

Note: 'Controlled for intervention group.

Abbreviations: $\mathrm{Cl}$, confidence interval; $\mathrm{OR}$, odds ratio; SD, standard deviation. 
(Lorenc and Branthwaite 1993; Salzman 1995). In a recent primary care study of older adults by Mackin and Areán (2007), cognitive functioning and depression severity were identified as predictors of missed appointments but not of treatment adherence.

Consistent with earlier findings from our research group, we found that nonadherence was predicted by sensation-seeking personality traits (Ekselius et al 2000). This finding is understandable, since individuals who are impulsive, nonplanning, and who avoid routines and have a need for change may not find it easy or significant to adhere to daily medication regimens and follow-up visits. The finding is also in line with another study reporting a strong association between novelty seeking personality traits and early discontinuation from clinical trials in patients with anxiety disorders (Wingerson et al 1993).

\section{Illness-related characteristics}

A somewhat unexpected finding in the present study was that patients with no concomitant medication were more likely to be nonadherent. Previous research on the impact of polypharmacy on adherence has mainly focused on the elderly. In a review by Vik and colleagues (2004) the authors concluded that "an increased number of medications may adversely affect adherence, although findings have not been consistent". A reasonable explanation for our finding may be that it is easier for patients with no other prescriptions to forget to take their medication. Another tentative explanation is that depressed patients with no other diseases, and consequently no other medications, have less insight into their illness and less belief in the benefits of treatment (especially when they start feeling better). Recent research has shown that the perceived stigma associated with mental illness and the individual patient's views about the illness and medication play an important role in adherence to treatment for depression (Brown et al 2005; Brook et al 2006; Mackin and Arean 2007). Furthermore, skepticism about antidepressants has been shown to predict early discontinuation of SSRIs (Aikens et al 2005). These findings are also in line with the extrapolated Health Belief Model, which states that individuals perform a cost/benefit analysis in which they determine if the benefits of treatment outweigh the costs (adverse effects, social stigma, cost of treatment, etc.) (Becker and Maiman 1975).

Substance abuse predicted nonadherence to antidepressant medication in the present study. In a recent study on medication adherence in first episode schizophrenia, alcohol abuse at baseline and previous drug abuse were shown to predict nonadherence (Kamali et al 2006). Further, in a study of psychiatrist-reported treatment nonadherence among patients in routine psychiatric care, substance abuse was shown to predict problems with adherence (Compton et al 2005).

Research concerning the impact of personality pathology on antidepressant adherence is sparse and somewhat conflicting. The present study revealed that the presence of a personality disorder increased the risk of nonadherence. In accord with this, Compton and colleagues (2005) found that a personality disorder diagnosis was associated with poor adherence. Analogously, Sirey and colleagues (2001) reported that medication adherence was predicted by the absence of personality pathology assessed by the Inventory of Interpersonal Problems. However, in a long-term study of prophylaxis in recurrent depression by Frank and colleagues (1992), no differences were observed between adherent and nonadherent patients with respect to the presence of personality disorder determined using the Personality Assessment Form.

\section{Methodological considerations}

A limitation of this study is that the SLICE study was not designed with the primary aim of confirming predictors of nonadherence. Moreover, it was not designed to explore the impact of emotional and psychological factors (eg, perceived stigma and the individual's views about the illness and medication), and such factors have recently been shown to affect adherence to antidepressant therapy (Sirey et al 2001; Brown et al 2005; Brook et al 2006). A tentative explanation regarding our results is that subjects in young or old age groups, with personality psychopathology, substance abuse or no other medications, present with such obstacles to adherence to a greater extent than those who are adherent.

A potential drawback of the study may be the classification of adherence versus nonadherence. The present study used a pre-defined definition of adherence; thus, patients who discontinued medication or withdrew from the study, regardless of the reason, were all classified as nonadherent. This approach might have resulted in an overestimation of nonadherence. Furthermore, no distinction was made between different types of nonadherent behavior. On the other hand, the aim of the study was to explore nonadherence, regardless of the reason, and to identify patient and illness characteristics that can be ascertained at treatment start. The ability to identify potential treatment failures, and then to give these patients special attention and take relevant action right from the start of treatment, may increase adherence to medication and the chance of recovery.

\section{Conclusion}

This study shows that certain patient- and illness-related characteristics may imply an increased risk of nonadherence 
to antidepressant treatment in primary care. Giving special attention to patients with such characteristics may improve adherence.

\section{Acknowledgments}

The study received financial support from Pfizer $A B$ and grants from the Swedish Research Council (K2007-62X20386-01-3: Finn Bengtsson; 15231: Lisa Ekselius).

\section{References}

Åkerblad AC, Bengtsson F, Ekselius L, et al. 2003. Effects of an educational compliance enhancement programme and therapeutic drug monitoring on treatment adherence in depressed patients managed by general practitioners. Int Clin Psychopharmacol, 18:347-54.

Åkerblad AC, Bengtsson F, von Knorring L, et al. 2006. Response, remission and relapse in relation to adherence in primary care treatment of depression: a 2-year outcome study. Int Clin Psychopharmacol, 21:117-24.

Aikens JE, Kroenke K, Swindle RW, et al. 2005. Nine-month predictors and outcome of SSRI antidepressant continuation in primary care. Gen Hosp Psych, 27:229-36.

[APA] American Psychiatric Association. 1994. Diagnostic and Statistical Manual of Mental Disorders (4th edn) (DSM-IV). Washington DC: APA.

Becker MH, Maiman LA. 1975. Sociobehavioral determinants of compliance with health and medical care recommendations. Med Care, 13:10-24.

Bengtsson F. 2004. Therapeutic drug monitoring of psychotropic drugs. TDM "nouveau”. Ther Drug Monit, 26:145-51.

Brook OH, van Hout HPJ, Stalman WAB, et al. 2006. Nontricyclic antidepressants. Predictors of nonadherence. J Clin Psychopharmacol, 26:643-7.

Brown C, Battista DR, Bruehlman R, et al. 2005. Beliefs about antidepressant medications in primary care patients. Med Care, 43:1203-7.

Bull SA, Hu XH, Hunkeler EM, et al. 2002. Discontinuation of use and switching of antidepressants: influence of patient-physician communication. JAMA, 18:1403-9.

Burke MJ, Preskorn SH. 1999. Therapeutic drug monitoring of antidepressants: cost implications and relevance to clinical practice. Clin Pharmacokinet, 37:147-65.

Cantrell CR, Eaddy MT, Shah MB, et al. 2006. Methods for evaluating patient adherence to antidepressant therapy: a real-world comparison of adherence and economic outcomes. Med Care, 44:300-3.

Cohen NL, Parikh SV, Kennedy SH. 2000. Medication compliance in mood disorders: relevance of the Health Belief Model and other determinants. Primary Care Psychiatry, 6:101-8.

Cohen NL, Ross EC, Bagby RM, et al. 2004. The 5-factor model of personality and antidepressant medication compliance. Can $J$ Psychiatry, 49:106-13.

Compton MT, Rudish BE, Weiss PS, et al. 2005. Predictors of psychiatristreported treatment-compliance problems among patients in routine US psychiatric care. Psychiatry Res, 137:29-36.

Demyttenaere K, Enzlin P, Dewé W, et al. 2001. Compliance with antidepressants in a primary care setting, 1: Beyond lack of efficacy and adverse events. J Clin Psychiatry, 62(Suppl 22):30-3.

Demyttenaere K, Van Ganse E, Gregoire J, et al. 1998. Compliance in depressed patients treated with fluoxetine or amitriptyline. Belgian Compliance Study Group. Int Clin Psychopharmacol, 13:11-7.

Demyttenaere K. 1997. Compliance during treatment with antidepressants. $J$ Affect Disord, 43:27-39.

Demyttenaere K. 2003. Risk factors and predictors of compliance in depression. Eur Neuropsychopharmacol, 13(Suppl):69-75.

Dolan P, Gudex C, Kind P, et al. 1996. The time trade-off method: results from a general population study. Health Econ, 5:141-54.
Ekselius L, Bengtsson F, von Knorring L. 2000. Non-compliance with pharmacotherapy of depression is associated with a sensation seeking personality. Int Clin Psychopharmacol, 15:273-8.

EuroQol Group. 1990. EuroQol: a new facility for the measurement of health-related quality of life. Health Policy, 16:199-208.

Frank E, Perel JM, Mallinger AG, et al. 1992. Relationship of pharmacologic compliance to long-term prophylaxis in recurrent depression. Psychopharmacol Bull, 28:231-5.

Gopinath S, Katon WJ, Russo JE, et al. 2007. Clinical factors associated with relapse in primary care patients with chronic or recurrent depression. $J$ Affect Disord, 101:57-63.

Gustavsson JP, Bergman H, Edman G, et al. 2000. Swedish universities Scales of Personality (SSP): construction, internal consistency and normative data. Acta Psychiatr Scand, 102:217-25.

Guy W. 1976. Clinical Global Impressions. In: ECDEU Manual. US Dept of Health and Human Services. Rockville MD: NIMH. pp. 217-22.

Hardin JW, Hilbe JM. 2002. Generalized Estimating Equations, Baton Rouge, FL: CRC Press.

Hosmer DW, Lemeshow S. 2000. Applied Logistic Regression. Hoboken, NJ: Wiley Inc.

Kamali M, Kelly BD, Clarke M, et al. 2006. A prospective evaluation of adherence to medication in first episode schizophrenia. Eur Psychiatry, 21:29-33.

Kutcher S, Leblanc J, Maclaren C, et al. 2002. A randomized trial of a specific adherence enhancement program in sertraline-treated adults with major depressive disorder in a primary care setting. Prog Neuropsychopharmacol Biol Psychiatry, 26:591-6.

Lin EH, Von Korff M, Katon W, et al. 1995. The role of the primary care physician in patients' adherence to antidepressant therapy. Medical Care, 33:67-74.

Lingam R, Scott J. 2002. Treatment non-adherence in affective disorders. Acta Psychiatr Scand, 105:164-72.

Lorenc L, Branthwaite A. 1991. Are older adults less compliant with prescribed medication than younger adults? Br J Clin Psychology, 32:485-92.

Mackin RS, Areán PA. 2007. Cognitive psychiatric predictors of medical treatment adherence among older adults in primary care clinics. Int $J$ Ger Psychiatry, 22:55-60.

Melfi CA, Chawla AJ, Croghan TW, et al. 1998. The effects of adherence to antidepressant treatment guidelines on relapse and recurrence of depression. Arch Gen Psychiatry, 55:1128-32.

Montgomery S, Åsberg M. 1979. A new depression scale designed to be sensitive to change. Br J Psychiatry, 134:382-9.

Mundt JC, Clarke GN, Burroughs D, et al. 2001. Effectiveness of antidepressant pharmacotherapy: the impact of medication compliance and patient education. Depress Anxiety, 13:1-10.

Olfson M, Marcus SC, Tedeschi M, et al. 2006. Continuity of antidepressant treatment for adults with depression in the United States. Am J Psychiatry, 163:101-8.

Osterberg L, Blaschke T. 2005. Adherence to medication. $N$ Engl J Med, 353:487-97.

Ottosson H, Bodlund O, Ekselius L, et al. 1998. DSM-IV and ICD-10 Personality Disorders: A comparison of a self-report questionnaire (DIP-Q) with a structured interview. Eur Psychiatry, 13:246-53.

Pampallona S, Bollini P, Tibaldi G, et al. 2002. Patient adherence in the treatment of depression. Br J Psychiatry, 180:104-9.

Salzman C. 1995. Medication compliance in the elderly. J Clin Psychiatry, 56(Suppl 1):18-22.

Sirey JA, Bruce ML, Alexopoulos GS, et al. 2001. Stigma as a barrier to recovery: Perceived stigma and patient-rated severity of illness as predictors of antidepressant drug adherence. Psychiatr Serv, 52:1615-20.

Vik SA, Maxwell CJ, Hogan DB. 2004. Measurement, correlates, and health outcomes of medication adherence among seniors. Ann Pharmacother, 38:303-12.

Wingerson D, Sullivan M, Dager S, et al. 1993. Personality traits and early discontinuation from clinical trials in anxious patients. $J$ Clin Psychopharmacol, 13:194-7. 